\title{
Research in neurodegenerative diseases: challenges and solutions
}

Kin Tam

Editor: $A D M E T \& D M P K$

E-mail: kin tam@iapchem.org

The need of effective medicines for the treatment of neurodegenerative diseases, such as Alzheimer's disease and Parkinson's disease, is expected to increase strongly in the coming decades. Though great efforts have been paid on research, neurodegenerative diseases remain as urgent unresolved problems in modern day medicine. At present, there is no cure for these diseases, resulting in death of neuron cells. Generally, the disease mechanisms are not fully understood and are thought to be related to genetics, protein misfolding/degradation or mitochondrial dysfunction etc. This introduces further complexity in the search for therapeutic approaches for these diseases.

In most neurodegenerative diseases, the blood-brain barrier (BBB) becomes a hurdle in effective drug delivery. Small molecules or biologics need to be engineered to cross the BBB and reach their target in sufficient concentrations to elicit a pharmacodynamics response. The drug concentration in the brain is tricky to determine, while behavioral assessment methods are used as surrogates for pharmacodynamics effects. In this context, it could be challenging to establish an unambiguous pharmacokinetics/pharmacodynamics relationship.

In this special issue, some papers reviewed the mechanisms, treatment approaches, animal models used for Alzheimer's disease. A paper reported a trend model for Alzheimer's disease mortality. Moreover, two review papers discussed BBB permeability and delivery in traumatic brain injury and in insulin delivery for Alzheimer's disease, respectively. Other papers dealt with cognitive deficits. Finally, two other papers discussed the use of nanotechnology platform in Parkinson's disease and the interactions of prion protein with metal ions.

For the first time, ADMET \& DMPK devotes a special issue on medical research/applications in a particular therapeutic area, with the aims to capture some of the current views and new achievements in neurodegenerative diseases. It is nice to see some papers discussing cross-disciplinary research and new thoughts to tackle these diseases. Hopefully, we will call for more special issues aligned to other therapeutic areas in due course. 